Çukurova Üniversitesi Mühendislik Mimarlık Fakültesi Dergisi, 35(1), ss. 211-219, Mart 2020

Çukurova University Journal of the Faculty of Engineering and Architecture, 35(1), pp. 211-219, March 2020

\title{
LiBr-Su Çözeltili Absorpsiyonlu Soğutma Çevriminin Soğutma Etkinliğinin Çeşitli Şartlarda Simülasyonu
}

\author{
Mehmet Tahir ERDINÇ ${ }^{1}$, Arif Emre AKTAŞ ${ }^{* 2}$, Alper YILMAZ ${ }^{2}$ \\ ${ }^{1}$ Tarsus Üniversitesi, Mühendislik Fakültesi, Makine Mühendisliği Bölümü, Tarsus \\ ${ }^{2}$ Çukurova Üniversitesi, Mühendislik Fakültesi, Otomotiv Mühendisliği Bölümü, Adana
}

Geliş tarihi: 30.01.2020 Kabul tarihi: 15.05.2020

\section{$\ddot{\mathbf{O z}}$}

İhmal edilebilir çevresel etkisi, oluşturduğu düşük gürültü ve titreşimi nedeniyle en çok tercih edilen sistemlerden biri absorpsiyonlu soğutma sistemleridir. Mekanik kompresörün bulunmadığı bu sistemlerde hareketli parça olarak sadece pompa bulunur. Günümüzde özellikle düşük dereceli atık 1sılarından yararlanılarak soğutma elde edilmesinde tercih edilir. Yüksek kapasiteli iklimlendirme sistemlerinde yaygın olarak kullanılırlar. Ayrıca gaz türbini atık ısısından yararlanmada bu tür soğutma sistemleri kullanılmaktadır. Düşük sıcaklıktaki uygun soğutma etkinliği katsayıları nedeniyle $\mathrm{LiBr}$ - Su sistemleri tercih edilmektedir. $\mathrm{Bu}$ çalışmada, LiBr-Su çözeltili absorpsiyonlu soğutma çevriminin bütün noktaları belirlenmiş ve çeşitli durumlara bağlı olarak soğutma etkinliğinin hesaplanması yapılmıştır. $50 \mathrm{~kW}$ 'lık bir sistem için, soğutma etkinliğinin soğutma suyu sicaklığı, isitma suyu sicaklı̆g hesaplanması yapılmıştır. Sistem etkinlik katsayısının 1sıtma suyu ve soğutulacak suyun sıcaklığı ile arttığı buna karşın soğutma suyu sıcaklığıyla azaldığı görülmüsşür. Ayrıca sistemdeki cihazların her birinin eşanjör analizi de yapılmıştır.

Anahtar Kelimeler: Atık 1sı, Soğutma, LiBr-su çözeltisi, Absorpsiyon soğutma

\section{Simulation of LiBr-Water Absorption Refrigeration Cycle Cooling Performance at Various Conditions}

\begin{abstract}
Absorption cooling systems are one of the most preferred systems due to their negligible environmental impact, low noise, and vibration. These systems without mechanical compressors only contain pumps as moving parts. Nowadays, it is especially preferred for obtaining cooling by using low grade waste heat. They are widely used in high capacity air conditioning systems. Such cooling systems are used to utilize the gas turbine waste heat. LiBr-Water systems are preferred due to their acceptable coefficient of performance at low temperatures. In this study, all points of the LiBr-Water absorption cooling cycle calculation are determined and cooling performance at various conditions is carried out. Cooling performance is calculated for a $50 \mathrm{~kW}$ system as a function of cooling water temperature, heating water temperature and cooled water temperature. It is observed that cooling performance of the system increases with the temperature of the heating water and the water to be cooled, but decreases with the cooling water temperature. In addition, heat exchangers in the system are also analyzed.
\end{abstract}

Keywords: Waste heat, Refrigeration, LiBr-Water solution, Absorption cooling

"Sorumlu yazar (Corresponding author): Arif Emre AKTAŞ, aeaktas@cu.edu.tr 


\section{GíRiş}

Güneş enerjisinin kullanıldığı soğutma teknolojileri özellikle yaz aylarında Akdeniz ikliminde geleneksel soğutma sistemlerinin yoğun elektrik gereksinimi nedeniyle son y1llarda yaygınlık göstermektedir. Akdeniz'e ek olarak özellikle Avrupa'nın güney kesimlerinde solar enerjili soğutma sisteminin kullanım oranı $\% 50$ 'lere ulaşmıştır [1,2]. Güneş kuşağ içinde yer alan ülkemizde de son 10 yılda güneş paneli kullanım sayısı yaklaşık 4 kat artmıştır [3].

İklimlendirme sistemlerinden biri olan olarak güneş enerjisi ile absorpsiyonlu soğutma üzerine çalışmalar birçok araştırmacı tarafından ayrıntılı olarak ele alınmıştır [4-7]. İklimlendirme sistemleri içerisinde absorpsiyonlu soğutma ile diğer çeşitli soğutma sistemleri Pridasawas [8] tarafından incelenmiștir. Bunlara basit tek etkili, çift etkili ve difüzyonlu absorpsiyon (Platen Munters) çevrimi örnek olarak verilebilir [8] Absorpsiyonlu soğutma sistemlerinin jeotermal santrallere entegre çalışmaları üzerine geniş bir araştırma Tesha [9] tarafindan gerçekleştirilmiștir. Güneş enerjisi ile soğutma teknolojileri hakkında genel bilgiler Büyükalaca ve Yılmaz [10] tarafindan açıklanmıştır. $\mathrm{LiBr}-\mathrm{H}_{2} \mathrm{O}$ sistemlerinin termodinamik hesapları hakkında ASHRAE [11] Klein ve Nellis [12], Florides ve arkadaşları [13]ve Herold ve arkadaşları [14] tarafindan temel bilgiler verilmiştir.

Absorpsiyonlu soğutma çevriminin termodinamik performansını arttırmaya yönelik birçok çalışma yapılmıştır. Martinho ve arkadaşları [15] tek etkili absorpsiyonlu soğutma çevriminin boyutsuz bir matematiksel modelini geliştirmişler ve deneysel sonuçlarla karşılaştırmışlardır. Chen ve arkadaşları [16] farklı akışkanların kullanıldığı absorpsiyonlu soğutma çevriminin termodinamik analizini yapmış ve $\mathrm{LiBr}-\mathrm{Su}$ kullanılan sistemden elde edilen COP'nin $\mathrm{Cu}_{2} \mathrm{Cl}_{5}$-Su kullanılandan daha yüksek olduğunu göstermişlerdir. Táboas ve arkadaşları [17] gemi motoru atık isısından yararlanan amonyağın soğutucu akışkan olarak kullanıldığ1 absorpsiyonlu soğutma çevrimini teorik olarak incelemişler ve 0,5 üzerinde COP değerleri elde etmişlerdir. Chen ve arkadaşları [18] ana parametre olarak emme basınç değeri ve basınç oranın kullanıldığı bir optimizasyon çalışması yapmıştır. Çalışmanın sonucunda optimum şartlardaki COP değerlerinin konvansiyonel çevrime göre \%16,7 kadar yükseltilebileceği görülmüştür. Farshi ve arkadaşları [19] ejektörün kullanıldığ1 absorpsiyonlu kombine çevrimi incelemişler ve tek etkili absorpsiyonlu çevrime göre kısmen daha yüksek COP değeri elde etmişlerdir. Özellikle daha düşük sıcaklıklarda bu farkın daha belirgin olduğu görülmüştür. Mohtaram ve arkadaşları [20] LiBr-su kullanılan absorpsiyonlu soğutma çevriminin ekserji analizini yapmış ve en büyük ekserji kaybının absorberde olduğu sonucuna ulaşmıştır. Liang ve arkadaşları [21] soğutucu akışkan olarak amonyağın kullanıldığı çift ejektörlü kombine absorpsiyonlu soğutma çevriminin termodinamik incelemesini numerik olarak yapmışlardır. Sıvı-buhar ejektör absorber girişine, buhar tahrikli ejektörü ise jeneratörün çıkışına yerleştirmişlerdir. Sonuç olarak 0,64 'lere varan COP değerlerine ulaşılmıştır. Cai ve arkadaşları [22] $\mathrm{NH}_{3}-\mathrm{NaSCN}$ and $\mathrm{NH}_{3}-\mathrm{LiNO}_{3}$ çözeltilerinin soğutucu akışkan olarak kullanıldığ 1 absorpsiyon soğutma sisteminin deneysel incelemesini yapmıştır. Sonuçlar $\mathrm{NH}_{3} \mathrm{NaSCN}$ çözeltisi kullanıldığında daha yüksek COP elde edildiğini ortaya koymuştur. Ozgoren ve arkadaşları [23] amonyak-su çözeltili solar absorpsiyonlu soğutma sisteminin saatlik performans analizini yapmıştır. Sistemin 3,5 kW'lık bir soğutma yükü için $35,95 \mathrm{~m}^{2}$ kollektör yüzey alanı bulunmuştur. Absorpsiyonlu soğutma sistemleri çeşitli kitaplarda ele alınmıştır [24-26].

$\mathrm{Bu}$ çalışmada $\mathrm{LiBr}-\mathrm{H}_{2} \mathrm{O}$ çözeltili soğutma sisteminin hesaplanması açıklanmış ve bilhassa kaynak sicak su ve elde edilen soğuk su sıcaklıklarının sistem performansına etkileri ayrıntılı bir şekilde tartışılmıştır. Sistemin gerçek soğutma etkinliği ve Carnot soğutma etkinliği arasındaki bağıntı araştırılmıştır. Ayrıca sistemdeki enerji transferi olan cihazların her birine ait eşanjör analizi de yapılmıştır. 


\section{2. ÇEVRIMIN AÇIKLANMASI}

İncelenen absorpsiyonlu soğutma sistemi Şekil 1'de şematik olarak gösterilmiştir. Sistemde $\mathrm{H}_{2} \mathrm{O}$ soğutucu akışkan ve LiBr de absorbe eden akışkan olarak kullanılmaktadır. Absorberden çıkan fakir çözelti, pompa tarafindan $\mathrm{P}_{\mathrm{e}}$ basıncından $\mathrm{P}_{\mathrm{k}}$ jeneratör basıncına yükseltilmektedir. Is1 değiştiricisinde sıcaklığı yükseltilerek 3 noktasında jeneratöre iletilmektedir. Burada çözelti isıtılmakta ve bir kısım su çözeltiden buharlaşarak kondensere gitmektedir. Bundan dolayı çözelti $\mathrm{LiBr}$ bakımından zenginleşerek 4 noktasına gelmektedir. LiBr bakımından zengin çözelti 1S1 değiştiricisi vasıtasıyla 1sısının bir kısmını fakir çözeltiye aktarmakta ve genleşme vanasında $\mathrm{P}_{\mathrm{e}}$ basıncına düşürülerek 6 noktasından absorbere iletilmektedir. Kondensere 7 noktasindan gelen su buharı burada $P_{k}$ basıncinda yoğuşmakta ve doymuş su da genleşme vanası üzerinden 9 noktasından evaporatöre iletilmektedir. Evaporatörde su buharlaşarak soğutması istenen suyu soğutmakta ve doymuş buhar olarak absorbere gelerek çözelti tarafından emilmektedir. Fakirleşen çözelti pompa tarafindan üst $\mathrm{P}_{\mathrm{k}}$ basıncına çıkarılarak çevrim tamamlanmaktadır. Absorpsiyon esnasındaki 1si da soğutma suyu tarafından alınmaktadır.

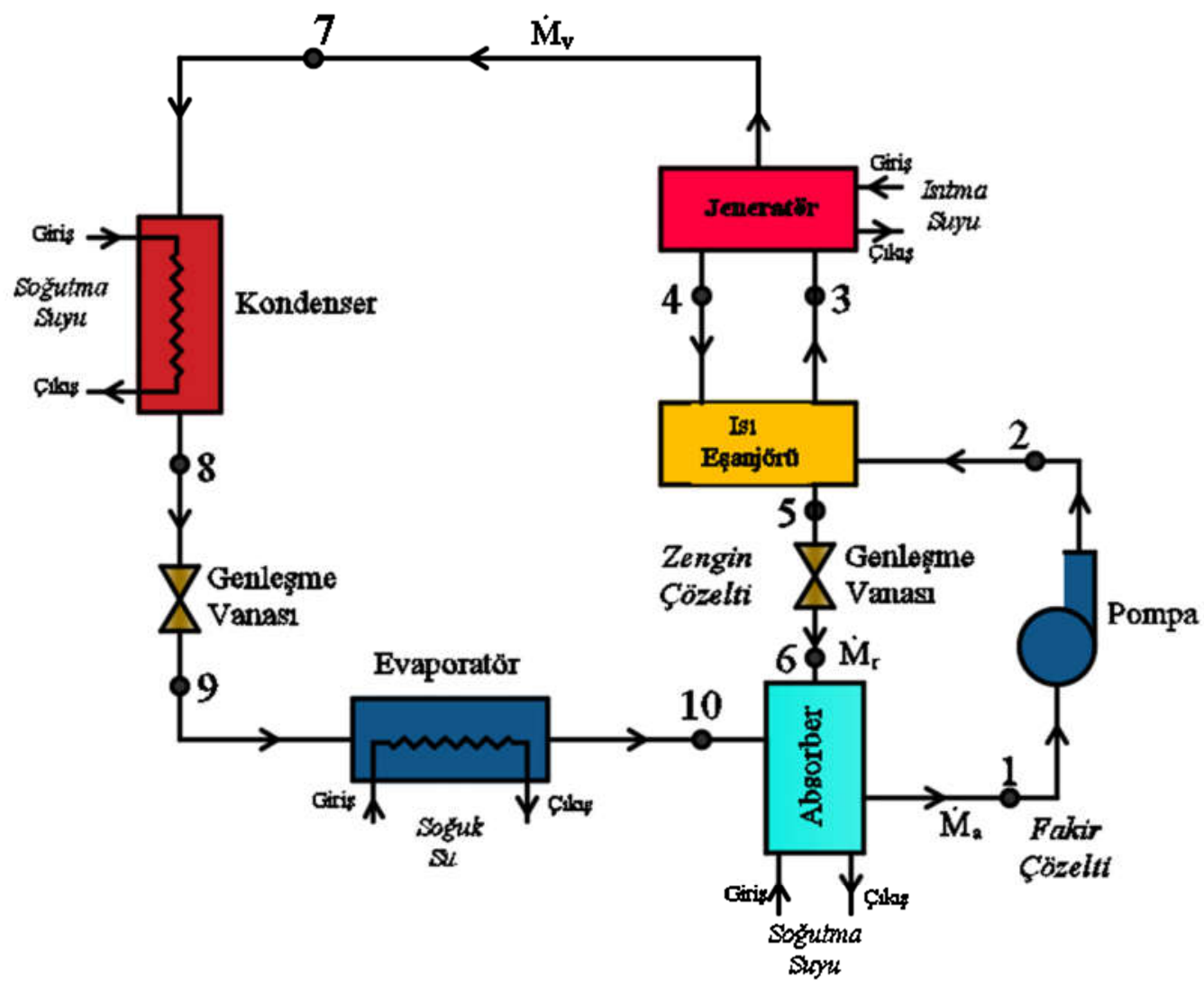

Şekil 1. $\mathrm{LiBr}-\mathrm{H}_{2} \mathrm{O}$ soğutma makinesinin şematik gösterimi

\section{TERMODINAMIK HESAPLAR}

$\mathrm{Bu}$ soğutma çevriminin hesaplanabilmesi için gerekli eşitlikler, ısı ve kütle bilançoları ve cihazlardan çıkış durumları için yapılacak varsayımlardan yararlanılarak elde edilmiştir. Termodinamik model şu varsayımlar göz önünde bulunarak yapılmıştır: 
(i) Is1 ve sürtünme kayıpları ihmal edilmiştir.

(ii) Kararlı durum şartları kabul edilmiştir.

(iii) Potansiyel ve kinetik enerji değişimi ihmal edilmiştir.

Pompadaki enerji bilançosu fakir eriyik debisi $\dot{M}_{a}$ ile şeklindedir (Eşitlik 1).

$$
\dot{M}_{a}\left(h_{2}-h_{1}\right)=\dot{W}_{p}
$$

$\dot{W}_{p}$ ise pompa izentropik verimi $\eta_{p}$ dikkate alınarak Eşitlik 2 yardımı ile hesaplanır.

$\dot{W}_{p}=\frac{\dot{M}_{a}\left(P_{k}-P_{e}\right)}{\rho_{1} \eta_{p}}$

Isı eşanjöründeki enerji bilançosundan da $\dot{M}_{r}$ zengin eriyik debisi ile elde edilir (Eşitlik 3).

$$
\dot{M}_{a}\left(h_{3}-h_{2}\right)=\dot{M}_{r}\left(h_{4}-h_{5}\right)
$$

Is1 eşanjörü etkinliği olarak $\varepsilon_{e}$ kabul edildiğinde de 2, 4 ve 5 noktalarındaki sıcaklıklar için Eşitlik 4'teki gibi yazılabilir:

$\mathrm{T}_{5}=\varepsilon_{\mathrm{e}} \cdot \mathrm{T}_{2}+\left(1-\varepsilon_{\mathrm{e}}\right) \mathrm{T}_{4}$

Jeneratöre verilen isı $\dot{Q}_{g}$ de, genleşme vanasından iletilen akışkan debisi $\dot{M}_{r}$ ile şeklinde elde edilir [24] (Eşitlik 5).

$\dot{\mathrm{Q}}_{\mathrm{g}}=\dot{\mathrm{M}}_{\mathrm{r}} \mathrm{h}_{4}+\dot{\mathrm{M}}_{\mathrm{v}} \mathrm{h}_{7}-\dot{\mathrm{M}}_{\mathrm{a}} \mathrm{h}_{3}$

Burada $\dot{M}_{v}$ ve $\dot{M}_{a}$ sirasıyla kondensere iletilen ve 1s1 eşanjöründen jeneratöre geçen akışkan debileridir. $\dot{Q}_{g}$ aynı zamanda aşağıdaki eşitlik yardımıyla da hesaplanır (Eşitlik 6).

$\dot{\mathrm{Q}}_{\mathrm{g}}=\dot{\mathrm{M}}_{\mathrm{h}} \cdot \mathrm{c}_{\mathrm{ph}}\left(\mathrm{T}_{\mathrm{hg}}-\mathrm{T}_{\mathrm{hc}}\right)$

Burada $c_{p h}$ sicak suyun özgül 1sisıdır. $\dot{M}_{h}$ sicak suyun kütlesel debisi ve $T_{h g}$ ve $T_{h c}$ ise sicak su giriş ve çıkış sıcaklıklarıdır (Eşitlik 7 ve 8). Jeneratördeki kütle

$$
\dot{\mathrm{M}}_{\mathrm{v}}=\dot{\mathrm{M}}_{\mathrm{a}}-\dot{\mathrm{M}}_{\mathrm{r}}
$$

$\dot{\mathrm{M}}_{\mathrm{a}} \mathrm{x}_{\mathrm{a}}=\dot{\mathrm{M}}_{\mathrm{r}} \mathrm{x}_{\mathrm{r}}$

bağıntıları yardımıyla elde edilir. Burada buhar içindeki LiBr ihmal edilmiştir. Bu kütle bilançoları absorber için de geçerlidir. Absorberdeki enerji bilançosundan aşağıdaki denklem elde edilir (Eşitlik 9):

$\dot{\mathrm{M}}_{\mathrm{r}} \mathrm{h}_{6}+\dot{\mathrm{M}}_{\mathrm{v}} \mathrm{h}_{10}=\dot{\mathrm{M}}_{\mathrm{a}} \mathrm{h}_{1}+\dot{\mathrm{Q}}_{\mathrm{a}}$

Absorberde atılan 1S1 $\dot{Q}_{a}$ için ayrıca eşitliği yazılabilir (Eşitlik 10).

$\dot{\mathrm{Q}}_{\mathrm{a}}=\dot{\mathrm{M}}_{\mathrm{ca}} \cdot \mathrm{c}_{\mathrm{p}_{\mathrm{ca}}}\left(\mathrm{T}_{\mathrm{cac}}-\mathrm{T}_{\mathrm{cag}}\right)$

Burada $\dot{M}_{c a}$ absorbere verilen soğutma suyu kütlesel debisi, $c_{p_{c a}}$ da bu suyun özgül 1sısıdır. $T_{\text {cag }}$ ve $T_{\text {caç }}$ sıcaklıkları da bu suyun giriş ve çıkış sıcaklıklarıdır.

Bunların yanısıra, genleşme vanasındaki proses izentalp (sabit entalpi) kabul edildiğinden eşitliği geçerlidir (Eşitlik 11).

$\mathrm{h}_{6}=\mathrm{h}_{5}$

Soğutucu akışkan devresinde ise kondenserdeki enerji bilançosundan yazılır (Eşitlik 12).

$\dot{\mathrm{Q}}_{\mathrm{k}}=\dot{\mathrm{M}}_{\mathrm{v}}\left(\mathrm{h}_{7}-\mathrm{h}_{8}\right)$

Ayrica, kondenserde soğutucu suya verilen 1S1 da aşağıdaki eşitlik yardımıyla hesaplanabilir (Eşitlik 13):

$\dot{\mathrm{Q}}_{\mathrm{k}}=\dot{\mathrm{M}}_{\mathrm{ck}} \cdot \mathrm{c}_{\mathrm{p}_{\mathrm{ck}}}\left(\mathrm{T}_{\mathrm{ckc}}-\mathrm{T}_{\mathrm{ckg}}\right)$

$\mathrm{Bu}$ eşitlikte $\dot{M}_{c k}$ kondensere verilen soğutma suyunun kütlesel debisi, $c_{p_{c k}}$ ise bu suyun özgül 1sısıdır. $T_{c k c ̧}$ ve $T_{c k g}$ sıcaklıkları da bu suyun çıkış ve giriş sıcaklıklarıdır. 
Genleşme vanasındaki durum değişimi izentalp kabul edildiğinden Eşitlik 14 yazılabilir.

$\mathrm{h}_{9}=\mathrm{h}_{8}$

Evaporatördeki enerji bilançosu yardımıyla yazılır (Eşitlik 15).

$\dot{\mathrm{Q}}_{\mathrm{e}}=\dot{\mathrm{M}}_{\mathrm{v}}\left(\mathrm{h}_{10}-\mathrm{h}_{9}\right)$

Ayrıca evaporatördeki soğutulan su kütlesel debisi $\dot{M}_{c e}$ ile de Eşitlik 16'daki gibi yazılabilir:

$\dot{Q}_{e}=\dot{M}_{c e} \cdot c_{p_{c e}}\left(T_{c e g}-T_{c e c ̧}\right)$

Bu eşitlikteki $c_{p_{c e}}$ bu suyun özgül 1sısıdır. $T_{c e g}$ ve $T_{\text {ceç }}$ sıcaklıkları ise bu suyun giriş ve çıkış sıcaklıklarıdır.

Sistemin analiz edilebilmesi için bazı noktalarda akışkan durumları için varsayımlar yapılmalıdır. Buna göre; kondenser ve jeneratördeki $P_{k}$ basınc1 eşit kabul edilmiştir. Su saf su ve 8 noktasındaki durumu ise doymuş sıvı kabul edilerek Eşitlik 17'deki gibi yazılır.

$\mathrm{P}_{\mathrm{k}}=\mathrm{f}\left(\mathrm{T}_{8}\right)$

Burada $T_{8}$ de kondenser soğutma suyu giriş sıcaklığına bağlı olup aşağıdaki eşitliğin geçerli olduğu kabul edilmiştir [25] (Eşitlik 18):

$$
T_{8}=T_{c k g}+8
$$

Ayrıca, evaporatör tarafındaki basınç $P_{e}$ de $T_{10}$ da doymuş buhar varsayımıyla hesaplanmıştır (Eşitlik 19):

$P_{e}=f\left(T_{10}\right)$

$T_{10}$ sıcaklığı ise, evaporatörde soğutulacak suyun giriş sıcaklığından aşağıdaki eşitlik vasıtasıyla elde edilir [25] (Eşitlik 20).
$\mathrm{T}_{10}=\mathrm{T}_{\text {ceg }}-8$

$T_{1}$ ve $T_{4}$ sıcaklıkları da jeneratör ve absorber giriş sıcaklıklarının fonksiyonu olarak aşağıdaki gibi kabul edilir [25] (Eşitlik 21 ve 22):

$\mathrm{T}_{1}=\mathrm{T}_{\text {cag }}+8$

$T_{4}=T_{h g}-8$

Çözelti entalpileri ise 1-6 noktaları arasındaki bu noktalardaki sicaklıklar ve konsantrasyon $\mathrm{x}$ 'in fonksiyonu olarak aşağıdaki gibi hesaplanır (Eşitlik 23):

$\mathrm{h}_{\mathrm{i}}=\mathrm{f}\left(\mathrm{T}_{\mathrm{i}}, \mathrm{x}_{\mathrm{i}},(\mathrm{LiBr}-\mathrm{Su})\right)$

7, 8 ve 10 noktalarındaki entalpiler de şeklinde hesaplanabilir (Eşitlik 23).

$\mathrm{h}_{\mathrm{i}}=\mathrm{f}\left(\mathrm{T}_{\mathrm{i}}, \mathrm{x}_{\mathrm{i}},(\mathrm{Su})\right)$

Ancak burada 7 ve 10 noktalarında doymuş buhar varsayımından dolayı $\mathrm{x}=1$ ve 8 noktasinda da doymuş su varsayımından dolayı $\mathrm{x}=0$ olarak alınır. Ayrıca 9 noktasındaki sicaklık denkleminden (Eşitlik 25),

$T_{9}=f\left(P_{e}, h_{9}\right)$

kuruluk derecesi de bağıntısından hesap edilebilir (Eşitlik 26).

$\mathrm{x}_{9}=\mathrm{f}\left(\mathrm{P}_{\mathrm{e}}, \mathrm{h}_{9}\right)$

Sistem soğutma etkinliği $C O P_{S}$ de eşitliğinden hesaplanırken (Eşitlik 27),

$\mathrm{COP}_{\mathrm{s}}=\frac{\dot{\mathrm{Q}}_{\mathrm{e}}}{\dot{\mathrm{Q}}_{\mathrm{g}}}$

Carnot etkinliği $[24,26]$ (Eşitlik 28), bağıntısından hesaplanabilir. 


$$
C O P_{C}=\left[1-\left(\frac{\mathrm{T}_{\mathrm{ckg}}}{T_{h g}}\right)\right] \cdot\left[\frac{\mathrm{T}_{\mathrm{ceg}}}{T_{c k g}-T_{\text {ceg }}}\right]
$$

\section{EŞANJÖR ANALİZI}

Örnek hesaplamalara esas alınan çevrim değerleri Çizelge 1'de ve hesaplanan sonuçlar da Çizelge 2'de verilmiştir. Hesaplamalarda kullanılan tüm 1sı eşanjörleri boru demeti şeklinde ve hem içten hem de diştan tek geçişli ve karşıt akışlı olarak kabul edilmiştir. Bundan dolayı da Eşitlik 29'a göre hesaplanır.

$\dot{\mathrm{Q}}=\mathrm{U} F \Delta \mathrm{T}_{\mathrm{m}}$

Burada $U$ ve $F$ sirasiyla toplam 1s1 transfer katsayısı ve toplam ısı transfer yüzey alanıdır. Cihazlardaki logaritmik ortalama sicaklık değerleri $\Delta T_{m}$ 'ler aşağıdaki gibi yazılabilir [14] (Eşitlik 30-34):

$$
\begin{aligned}
& \Delta T_{\text {ma }}=\frac{\left(\mathrm{T}_{6}-\mathrm{T}_{\text {cac }}\right)-\left(\mathrm{T}_{1}-\mathrm{T}_{\text {cag }}\right)}{\ln \frac{\mathrm{T}_{6}-\mathrm{T}_{\text {caç }}}{\mathrm{T}_{1}-\mathrm{T}_{\text {cag }}}} \\
& \Delta T_{m k}=\frac{\left(T_{8}-T_{\text {ckg }}\right)-\left(T_{8}-T_{\text {ceç }}\right)}{\ln \frac{T_{8}-T_{\text {ckg }}}{T_{8}-T_{\text {ceç }}}} \\
& \Delta T_{m g}=\frac{\left(T_{h g}-T_{4}\right)-\left(T_{h c ̧}-T_{7}\right)}{\ln \frac{T_{h g}-T_{4}}{T_{h c ̧}-T_{7}}} \\
& \Delta T_{\text {me }}=\frac{\left(T_{\text {ceg }}-T_{10}\right)-\left(T_{\text {ceç }}-T_{9}\right)}{\ln \frac{T_{\text {ceg }}-T_{10}}{T_{\text {ceç }}-T_{9}}} \\
& \Delta T_{\text {mes }}=\frac{\left(T_{4}-T_{3}\right)-\left(T_{5}-T_{2}\right)}{\ln \frac{T_{4}-T_{3}}{T_{5}-T_{2}}}
\end{aligned}
$$

UF değerleri de Eşitlik (35-39)'daki gibi hesaplanırlar.

$$
\mathrm{UF}_{\mathrm{a}}=\frac{\dot{\mathrm{Q}}_{\mathrm{a}}}{\Delta \mathrm{T}_{\mathrm{ma}}}
$$

$$
\begin{aligned}
& \mathrm{UF}_{\mathrm{k}}=\frac{\dot{\mathrm{Q}}_{\mathrm{k}}}{\Delta \mathrm{T}_{\mathrm{mk}}} \\
& \mathrm{UF}_{\mathrm{e}}=\frac{\dot{\mathrm{Q}}_{\mathrm{e}}}{\Delta \mathrm{T}_{\mathrm{me}}}
\end{aligned}
$$

$\mathrm{UF}_{\mathrm{g}}=\frac{\dot{\mathrm{Q}}_{\mathrm{g}}}{\Delta \mathrm{T}_{\mathrm{mg}}}$

$\mathrm{UF}_{\mathrm{es}}=\frac{\dot{\mathrm{Q}_{\mathrm{g}}}}{\Delta \mathrm{T}_{\mathrm{mes}}}$

Çizelge 1'de eşanjör analizi için hesaplamalarda kullanılan parametreler verilmiştir.

Çizelge 1. Eşanjör hesabı için verilen değerler

\begin{tabular}{|c|c|}
\hline$\dot{\mathrm{Q}}_{\mathrm{e}}=50 \mathrm{~kW}$ & $\mathrm{~T}_{\mathrm{hg}}=90$ \\
\hline$\dot{\mathrm{M}}_{\mathrm{wh}}=2,5$ & $\mathrm{~T}_{\mathrm{ckg}}=31$ \\
\hline$\dot{\mathrm{M}}_{\mathrm{ca}}=2,5$ & $\mathrm{~T}_{\mathrm{cag}}=31$ \\
\hline$\dot{\mathrm{M}}_{\mathrm{ce}}=2,5$ & $\mathrm{~T}_{\mathrm{ceg}}=12$ \\
\hline$\dot{\mathrm{M}}_{\mathrm{ck}}=2,5$ & $\mathrm{~T}_{\mathrm{cec}}=8$ \\
\hline$\eta_{\mathrm{p}}=0,7$ & $\varepsilon_{\mathrm{e}}=0,7$ \\
\hline
\end{tabular}

\section{SAYISAL SONUÇLAR}

Örnek sayısal sonuçlar için soğutma kapasitesi $\dot{Q}_{e}=50 \mathrm{~kW}$, soğutma suyu ve soğutulacak su debileri de $2,5 \mathrm{~kg} / \mathrm{s}$ alınarak hesaplar yapılmıştır. Eşanjör etkinliği ve pompa verimi \%70 olarak kabul edilmiştir. $\mathrm{Bu}$ durumda sicak su giriş, soğutma suyu giriș ve soğutulacak su giriș sıcaklıkları değiştirilerek COP'lerin değişimi Şekil 2, 3 ve 4'te verilmiştir.

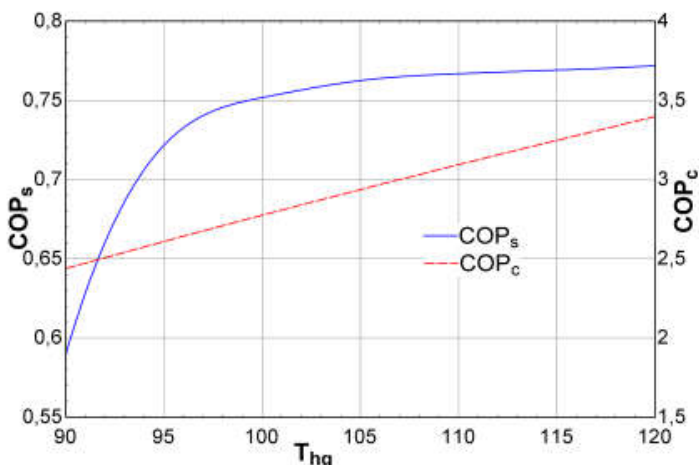

Şekil 2. COP'lerin $T_{h g}$ ile değiş̧imi 
Şekil 2'de COP değerlerinin jeneratöre giren sıcak su sıcaklığı $T_{\text {hg }}$ 'ye bağlı değişimi $T_{\text {ckg }}=31{ }^{\circ} \mathrm{C}$ ve $\mathrm{T}_{\text {ceg }}=12{ }^{\circ} \mathrm{C}$ için gösterilmiştir. Şekilde görüldüğü gibi, $\mathrm{COP}_{\mathrm{s}} 90{ }^{\circ} \mathrm{C}$ den sonra hizla artmakta ve $100{ }^{\circ} \mathrm{C}$ den sonra da bu artış yavaşlamaktadır. Buna karşın $\mathrm{COP}_{\mathrm{c}}$ 'nin doğrusal bir şekilde arttığı görülmektedir.

Şekil 3'te COP'lerin evaporatöre giren soğutma suyu sıcaklığına bağlı değişimi $\mathrm{T}_{\mathrm{hg}}=90{ }^{\circ} \mathrm{C}$ ve $\mathrm{T}_{\text {ceg }}=12{ }^{\circ} \mathrm{C}$ için verilmiştir. Burada kondenser ve absorberin soğutması için kullanılan soğutma suyunun sıcaklığı düşürüldüğünde $\mathrm{COP}_{\mathrm{s}}$ ve $\mathrm{COP}_{\mathrm{c}}{ }^{\prime}$ nin arttı̆̆ açıkça görülmektedir. $\mathrm{COP}_{\mathrm{s}}$ yüksek sıcaklıklarda daha hızlı değişirken $\mathrm{COP}_{\mathrm{c}}$ düşük sıcaklıklarda bu hızlı davranışı sergilemektedir. $\mathrm{Bu}$ değişim $\mathrm{COP}_{\mathrm{S}}$ için $\% 40,27$ iken $\mathrm{COP}_{\mathrm{c}}$ için \%181,79 mertebesindedir.

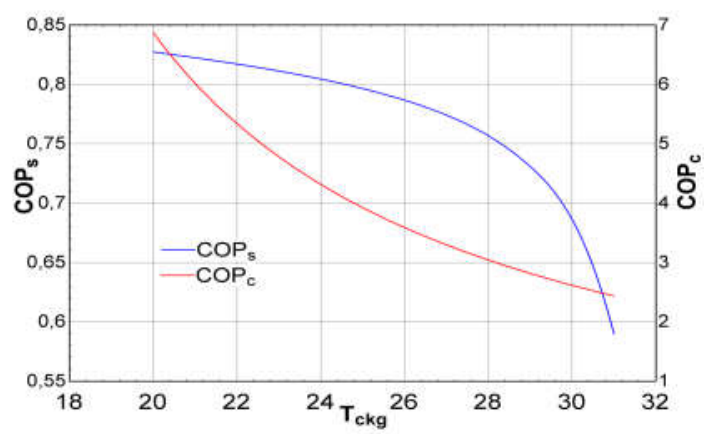

Şekil 3. COP'lerin $T_{\text {ckg }}$ ile değişimi

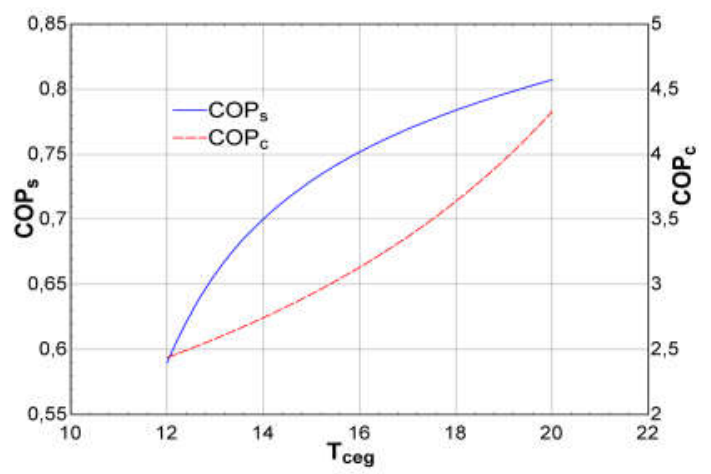

Şekil 4. COP'lerin $T_{\text {ceg }}$ ile değişimi

Şekil 4'te ise evaporatörde soğutulacak su giriş sıcaklığının COP'ye etkisi $\mathrm{T}_{\text {ckg }}=31 \quad{ }^{\circ} \mathrm{C}$ ve
$\mathrm{T}_{\mathrm{hg}}=90^{\circ} \mathrm{C}$ için gösterilmiştir. Buradan görüleceği gibi $\mathrm{COP}_{\mathrm{s}}$, soğutulacak su sıcaklığı arttıkça önce hızlı daha sonra da daha yavaş şekilde artmakta iken $\mathrm{COP}_{\mathrm{c}}$ bunun tersi bir artış göstermektedir. $\mathrm{Bu}$ artış $\mathrm{COP}_{\mathrm{s}}$ için \%36,86 iken $\mathrm{COP}_{\mathrm{c}}$ için \% 77,56 'tür. Her üç şekil karşıllaştırıldığında COP'yi en çok etkileyen parametrenin evaporatör sıcaklığı olduğu görülmektedir.

Çizelge 3. Cihazların $\dot{Q}$ ve $\Delta T_{m}$ değerleri

\begin{tabular}{|c|c|c|}
\hline Cihaz & $\dot{\boldsymbol{Q}}[\mathbf{k W}]$ & $\Delta \boldsymbol{T}_{\boldsymbol{m}}{ }{ }^{\mathbf{0}} \mathbf{C} \boldsymbol{]}$ \\
\hline Jeneratör & 67.41 & 18.05 \\
\hline Kondenser & 51.36 & 5.164 \\
\hline Evaporatör & 50.00 & 5.098 \\
\hline Absorber & 66.05 & 8.518 \\
\hline Is1 Eşanjörü & 19.47 & 16.54 \\
\hline
\end{tabular}

Çizelge 1'deki verilere göre belirlenen $\Delta T_{m}$ ve UF değerleri Çizelge 2'de tüm cihazlar için verilmiştir. Burada en yüksek 1s1 transferinin jeneratör ve absorberde olduğu anlaşılmaktadır. Şekil 5 'te ise bu verilerden türetilen UF değerleri verilmiştir. Bu değerler jeneratör, kondenser, evaporatör, absorber ve 1S1 eşanjörü için sırasıyla $3,735,9,947,9,808$, 7,754 ve $1,177 \mathrm{~W} /{ }^{\circ} \mathrm{C}^{\prime}$ dir.

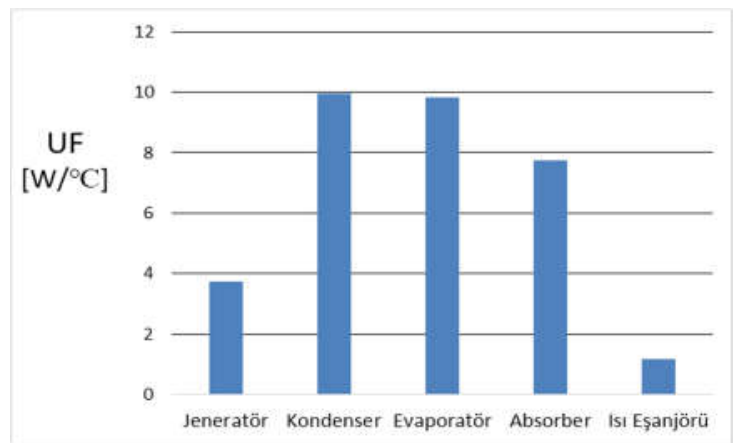

Şekil 5. Cihazların UF değerleri

\section{SONUÇLAR}

$\mathrm{Bu}$ çalı̧̧mada LiBr-Su çözeltisinin akışkan olarak kullanıldığı bir absorpsiyonlu soğutma makinesinin simülasyonu yapılmış ve sistemin tüm parametreleri ile sistemin soğutma etkinliği $\mathrm{COP}_{\mathrm{s}}$ ile karnot etkinliği $\mathrm{COP}_{\mathrm{c}}$ 'nin değişimi hesaplanmıştır. Çalışmada, sistemin soğutma 
etkinliklerinin sicak su, soğutma suyu ve soğutulacak su sıcaklıklarından nasıl etkilendiği şekillerle açıklanmıştır. Ayrıca sistemdeki cihazların her birindeki UF değerleri bulunmuştur. Buradan şu sonuçlar çıkarılabilir.

- COPs jeneratörde kullanılan sicak su sicaklığ $\mathrm{T}_{\text {hg }}$ ve evaporatörde kullanılan soğutma suyu $\mathrm{T}_{\text {ceg }}$ ile artmakta, kondenserde kullanilan soğutulacak su $\mathrm{T}_{\text {ckg }}$ ile azalmaktadır. $\mathrm{COP}_{\mathrm{c}}$ ise $\mathrm{T}_{\text {hg }}$ ve $\mathrm{T}_{\text {ceg }}$ ile artmakta $\mathrm{T}_{\text {ckg }}$ ile azalmaktadır.

- Jeneratörde kullanılan sicak su sicaklığ1 $\mathrm{T}_{\mathrm{hg}}$ 90 C'den $105{ }^{\circ} C^{\prime}$ ye çıkarken COPs artışı $\% 29,3$ iken, $105{ }^{\circ} \mathrm{C}$ 'den $120{ }^{\circ} \mathrm{C}$ 'ye çıkarken bu artış \%1,23 olmaktadır.

- Kondenserde kullanılan su sicaklığ $\mathrm{T}_{\text {ckg }}$ $21{ }^{\circ} \mathrm{C}$ 'den $26{ }^{\circ} \mathrm{C}$ 'ye çıkarken COPs'deki düşüş $\% 4,35$ iken, $26{ }^{\circ} \mathrm{C}^{\prime}$ den $31{ }^{\circ} \mathrm{C}^{\prime}$ ye çıkarken bu düşüş büyümekte ve $\% 25$ seviyelerine ulaşmaktadır.

- Evaporatörde kullanılan soğutma suyu sıcaklığı $\mathrm{T}_{\text {ceg }} 12{ }^{\circ} \mathrm{C}$ 'den $16{ }^{\circ} \mathrm{C}$ 'ye çıkarken COPs artış1 $\% 27,5$ iken, $16{ }^{\circ} \mathrm{C}^{\prime}$ den $20{ }^{\circ} \mathrm{C}^{\prime}$ ye çıkarken bu artış \% 7,6 olmaktadır.

- En yüksek UF değerinin kondenserde olduğu tespit edilmiştir.

\section{KAYNAKLAR}

1. Balaras, C.A., Grossman G., Henning, H.M., Ferreira, C.A.I., Podesser, E., Wang, L., Wiemken, E., 2007. Solar Air Conditioning in European Overview, Renewable and Sustainable Energy Reviews 11, 299-314.

2. Galindo, J., Dolz, V., García-Cuevas, L.M., Ponce-Mora, A., 2020. Numerical Evaluation of a Solar-Assisted Jet-Ejector Refrigeration System: Screening of Environmentally Friendly Refrigerants. Energy Conversion Management. 210, 112681.

3. Çanka, F., 2015. Solar Energy, its Recent Status in Turkey and Production Technologies, Mühendis ve Makina, 56(671), 28-40.

4. Kim, D.S., Infante Ferreira, C.A., 2008. Solar Refrigeration Options-a State-of-the-art Review, International Journal of Refrigeration, 31(1), 3-15.
5. Li, Z., Sumathy, K., 2000. Technology Development in the Solar Absorption airConditioning Systems, Renewable and Sustainable Energy Reviews, 4(3), 267-293.

6. Lamp, P., Ziegler, F., 1998. European Research on Solar-Assisted Air Conditioning, International Journal of Refrigeration, 21(2), 89-99.

7. Grossman, G., 2002. Solar-Powered Systems for Cooling, Dehumidification and AirConditioning, Solar Energy, 72(1), 53-62.

8. Pridasawas, W., 2006. Solar-Driven Refrigeration Systems with Focus on the Ejector Cycle, PhD Thesis, Royal Institute of Technology.

9. Tesha, T.P., 2009. Absorption Refrigeration System as an Integrated Condenser Cooling Unit in Geothermal Power Plant. Proceedings World Geothermal Congress, MSc Thesis, Department of Mechanical and Industrial Engineering.

10. Büyükalaca, O., Yılmaz, T., 2003. Güneş Enerjisi ile Soğutma Teknolojilerine Genel bir Bakış, Tesisat Mühendisliği, 45-56.

11. ASHRAE Handbook: Fundamentals. Thermodynamics and Refrigeration Cycles, American Society of Heating, Refrigeration and Air-Conditioning Engineers, 2009.

12. Klein, S.A., Nellis, G., 2012. Thermodynamics, Cambridge University Press.

13. Florides, G.A., Kalogirou, S.A., Tassou, S.A. Wrobel, L.C., 2003. Design and Construction of a $\mathrm{LiBr}-$ Water Absorption Machine, Energy Conversion and Management, 44(15), 2483-2508.

14. Herold, K.E., Radermacher, R., Klein, S.A., 2016. Absorption Chillers and Heat Pumps, 2nd ed. United Kingdom: Boca Raton, FL, CRC Press, Taylor \& Francis Group.

15. Martinho, L.C.S., Vargas, J.V.C. Balmant, W., Ordonez, J.C. 2016. A Single Stage Absorption Refrigeration System Dynamic Mathematical Modeling, Adjustment and Experimental Validation, Int. J. Refrig. 68, 130-144.

16. Chen, W., Bai, Y., 2016. Thermal Performance of an Absorption-Refrigeration System with $\mathrm{Cu}_{2} \mathrm{Cl}_{5} / \mathrm{NH}_{3}$ as Working Fluid, Energy, 112, 332-341. 
17. Táboas, F., Bourouis, M., Vallès, M., 2014. Analysis of Ammonia/Water and Ammonia/Salt Mixture Absorption Cycles for Refrigeration Purposes in Fishing Ships, Applied Thermal Engineering, 66 (1-2), 603-611.

18. Chen, Y., Han, W., Jin, H., 2016. Thermodynamic Performance Optimization of the Absorption-Generation Process in an Absorption Refrigeration Cycle, Energy Conversion Management, 126, 290-301.

19. Farshi L.G., Mosaffa, A.H., Infante Ferreira, C.A., Rosen, M.A., 2014. Thermodynamic Analysis and Comparison of Combined Ejector-Absorption and Single Effect Absorption Refrigeration Systems, Appl. Energy, 133, 335-346.

20. Mohtaram S., Omidi M., Lin J., Sun, H., Chen, W., 2019. Exergy Analysis of a Multi Mixture Working Fluid Absorption Refrigeration Cycle, Case Studies in Thermal Engineering, 15, 100540.

21. Liang, X., Zhou, S., Deng, J., He, G., Cai, D. 2019. Thermodynamic Analysis of a Novel Combined Double Ejector-Absorption Refrigeration System using Ammonia/Salt Working Pairs without Mechanical Pumps, Energy, 185, 895-909.

22. Cai, D., Jiang, J., He, G., Li, K., Niu, L., Xiao, R., 2016. Experimental Evaluation on Thermal Performance of an Air-Cooled Absorption Refrigeration Cycle with $\mathrm{NH}_{3}-\mathrm{LiNO}_{3}$ and $\mathrm{NH}_{3}-\mathrm{NaSCN}$ refrigerant Solutions, Energy Conversion Management 120, 32-43.

23. Ozgoren, M., Bilgili, M., Babayigit, O., 2012. Hourly Performance Prediction of Ammoniawater Solar Absorption Refrigeration, Appl. Therm. Engineering, 40, 80-90.

24. Çengel, Y.A., Boles, M.A., 2006. Thermodynamics an Engineering Approach, McGraw- Hill, USA, 1-865.

25. Yılmaz, T., 2016. Soğutma Teknolojisi, Osmaniye Korkut Ata Üniversitesi Ders Notlar1.

26. Yamankaradeniz, R., Horuz, İ., Coşkun, S., Kaynakl1, Ö., Yamankaradeniz., N., Soğutma Tekniği ve Isı Pompası Uygulamaları, 2017. Dora Basım Yayın, Bursa. 
